\title{
The spin of the nucleon in deep inelastic lepton scattering experiments
}

\author{
Gerhard K. Mallot \\ CERN, Division PPE, 1211 Geneva 23, Switzerland and \\ Mainz University, Inst. für Kernphysik, $6500 \mathrm{Mainz}$, Germany
}

\begin{abstract}
The current experimental status of the spin dependent structure functions as measured in polarised deep inelastic scattering of charged leptons from nucleons is reviewed. The proposals for new experiments at CERN, SLAC and HERA are discussed with special emphasis on the experiment of the Spin Muon Collaboration at CERN which bas started data taking.
\end{abstract}

\section{INTRODUCTION}

Since evidence for partons was found in the early SLAC-MIT' experiments [1], deep inelastic lepton scattering has been one of the cleanest tools to study the internal structure of the nucleon. While experiments measuring the spin independent structure functions have reached a high level of statistical and systematical accuracy for the proton as well as for the neutron, experiments using polarised targets and polarised beams had to overcome significant additional experimental difficulties. Up to now nothing is known experimentally on the two spin dependent structure functions $g_{1}^{n}$ and $g_{2}^{n}$ of the neutron and on $g_{2}^{p}$ of the proton. The furst information on the internal spin structure of the proton was obtained by the pioneering Yale-SLAC experiment (2). Their data were found to be in agreernent with the prediction of simple theoretical models. It came as a surprise when the European Muon Collaboration (EMC) at CERN announced a result indicating that only a negligible fraction of the proton spin is carried by its quarks and antiquarks (3). The unexpected result arises from a kinematic region unmeasured by the Yale-SLAC experiment. This effect, often referred to as the proton "spin crisis", has stimulated numerous new theoretical ideas and several proposals for new experiments. Presently the experimental input needed most, are the verification of the EMC result for the proton with higher statistical and systematical accuracy and the determination of $g_{1}$ for the neutron in order to test the fundamental Bjorken sum rule [4]. The new experiments will be discussed in sections 4 and 5 . For a recent review of polarised deep iselastic lepton scattering, see ref. [5].

$$
\text { 5b } 190
$$

Proceedings of the 13 th Intermational Conference on Few Body Problems in Physics

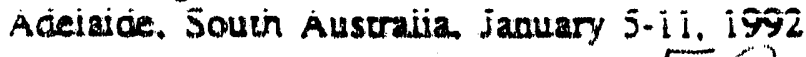




\section{POLARISED DEEP INELASTIC LEPTON SCATTERING}

In addition to the two spin independent structure functions $F_{1}$ and $F_{2}$ obserrable in unpolarised deep inelastic charged lepton scattering, two spin dependent structure functions, namely $g_{1}$ and $g_{2}$ can be studied in experiments using a polarised bearn and a polarised target. The cross section can be written as $\mathrm{d} \sigma=\mathrm{d} \bar{\sigma} \pm \mathrm{d} \Delta \sigma$, where $\mathrm{d} \bar{\sigma}$ denotes the unpolarised cross section depending on $F_{1}$ and $F_{2}$ and the sign depends on the helicity of the incident lepton. The spin dependent part $\mathrm{d} \Delta \sigma$ is given by [6]:

$$
\begin{aligned}
\frac{\mathrm{d} \Delta \sigma}{\mathrm{d} x \mathrm{~d} y \mathrm{~d} \phi}= & \frac{e^{4}}{4 \pi^{2} Q^{2}}\left\{\cos \alpha\left[\left(1-\frac{y}{2}-\frac{y^{2}}{4} \gamma^{2}\right) g_{1}\left(x, Q^{2}\right)-\frac{y}{2} \gamma^{2} g_{2}\left(x, Q^{2}\right)\right]\right. \\
& \left.-\gamma \sin \alpha \cos \phi \sqrt{1-y-\gamma^{2} \frac{y^{2}}{4}}\left(\frac{y}{2} g_{1}\left(x, Q^{2}\right)+g_{2}\left(x, Q^{2}\right)\right)\right\} .
\end{aligned}
$$

Here $\alpha$ is the angle between the direction of the incident lepton and the polarisation vector of the target, $\phi$ is the angle between the scattering plane and the plane defined by the incident lepton and the target polarisation vector, $y=\nu / E$ is relative energy transfer, $Q^{2}=-q^{2}$ the negative square of the four momentum transfer $q$ and $x=$ $Q^{2} / 2 . M \nu$ is the Bjorken scaling variable. The quantity $\gamma^{2}=Q^{2} / \nu^{2}$ approaches zero for $Q^{2} \rightarrow \infty$. Like $F_{1}$ and $F_{2}$ also $g_{1}$ and $g_{2}$ show scaling behaviour, i.e. $g\left(x, Q^{2}\right) \rightarrow g(x)$ for $Q^{2} \rightarrow \infty$. In the Quark-Parton Model $g_{1}$ has a simple and clear interpretation similar to the one of the structure function $F_{1}$

$g_{1}=\frac{1}{2} \sum e_{i}^{2}\left[q_{i}^{+}(x)-q_{i}^{--}(x)\right], \quad F_{l}=\frac{1}{2} \sum e_{i}^{2}\left[q_{i}^{+}(x)+q_{i}^{-}(x)\right]$,

where $q_{i}^{ \pm}$denotes the distribution function of the quarks of flavour $i$ and spin parallel or antiparallel to the nucleon spin, respectively. On the other hand a simple interpretation of $g_{2}$, which includes a twist-3 part measuring quark-gluon correlations [6], is not possible in the Quark-Parton Model.

Up to now all experiments were carried out with $\sin \alpha=0$. i.e. parallel or antiparallel orientation of target and lepton spin, where $g_{1}$ dominates and $g_{2}$ is suppressed by the factor $\gamma^{2}$. Transverse polarisation of the target spin allows a measurement of $g_{1}+g_{2}$ from which $g_{2}$ can be extracted.

Experimentally $g_{1}$ is extracted from the asymmetry $A$

$A=\frac{\mathrm{d} \sigma^{\dagger \downarrow}-\mathrm{d} \sigma^{\| 1}}{\mathrm{~d} \sigma^{\dagger \dagger}+\mathrm{d} \sigma^{\| 1}}=\frac{2 \mathrm{~d} \Delta \sigma}{2 \mathrm{~d} \bar{\sigma}}$

where $\uparrow \uparrow(\uparrow \downarrow)$ denotes parallel (antiparalle') longitudinal polarisation of the target spins with respect to the one of the incident lepton. Neglecting the contributions from $g_{2}$, which are small and bound by a positivity limit $[7]$, the structure funcion $g_{1}$ is given by

$g_{1}=\frac{A}{D} \cdot \frac{F_{2}}{2 x(1+R)}$ 
where the depolarisation factor $D$ depends only on the kinematics, and the ratio of the cross sections for longitudinal and transverse polarised virtual photons $R[8]$ and the structure function $F_{2}[9]$ are known from unpolarised experiments. The actually observed asymmetry $\Delta$

$\Delta=\frac{N^{1 !}-N^{1 \dagger}}{N^{1 !}+N^{\pi}}=P_{b} P_{t} f A$

is reduced from $A$ by the product of the beam polarisation $P_{b}$, the target polarisation $P_{t}$ and the dilution factor $f$, which is the fraction of polarisable nucleons in the target material. In the E.MC experiment $\Delta$ was of the order of $1 \%$.

\section{EARLIER RESULTS}

From the Yale-SLAC experiment [2], in which $23 \mathrm{GeV}$ electrons were scattered from a polarised butanol target and the EMC experiment at CERN [3], in which 100-200 GeV muons were scatiered from a polarised ammonia target, the spin dependent structure function $g_{1}^{p}$ of the proton is known in the range $0.01<x<0.7$. The data sets agree in the region of overlap mutually, however one should stress the importance of the low $x$ EMC data points for the evaluation of the integral of $g_{1}$ over $x$. The analysis of the combined data yields

$\Gamma^{p}=\int_{0}^{1} g_{1}^{p}(x) \mathrm{d} x=0.126 \pm 0.010 \pm 0.015$

for the first moment $\Gamma^{p}$ of $g_{1}^{p}$ with the quoted statistical and systematical errors (Fig. 1). This result is in significant disagreement with the Ellis-Jaffe sum rule [10] evaluated at the mean $Q^{2}$ of the EMC data of $10.7(\mathrm{GeV} / c)^{2}, \Gamma^{p}=0.189$. A more recent evaluation [i1) gives $\Gamma^{p}=0.175 \pm 0.018$. In the derivation of this sum rule flavour $\mathrm{SU}(3)$ symmetry in the baryon octet and an unpolarised strange sea are assumed. Giving up the latter, EMC extracted the fraction of the proton spin carried by quarks and antiquarks to be $\Delta \Sigma=12 \pm 9 \pm 14 \%$ compatible with zero. The contributions of the quarks and antiquarks of flavour up, down and strange were obtained as $78,-47$ and $-19 \%$ respectively, indicating a strange sea strongly polarised oppositely to the proton spin.

Provided the fundamental Bjorken sum rule $\Gamma^{p}-\Gamma^{n}=0.191$ is not violated, the EMC resuit for $\Gamma^{p}$ implies a value of $-0.065 \pm 0.025$ for $\Gamma^{n}$, the first moment of $g_{1}^{n}$ of the neutron. The corresponding value expected from the Ellis-Jaffe sum rule for the neutron is consistent with zero.

A review of the theoretical ideas attempting to understand the EMC result, is beyond the scope of this paper and excellent summaries exist (11). Only a short survey of the principal classes of arguments will be given. It has been suggested, that the Ellis-Jafie sum rule could still be saturated in the unmeasured very low $x$-region [12], but this would require an $x$-dependence of $g_{1}$ contradicting most theoretical expectations. A strong $Q^{2}$. dependence, which has been proposed as an explanation for the EMIC result [13], jeerns 


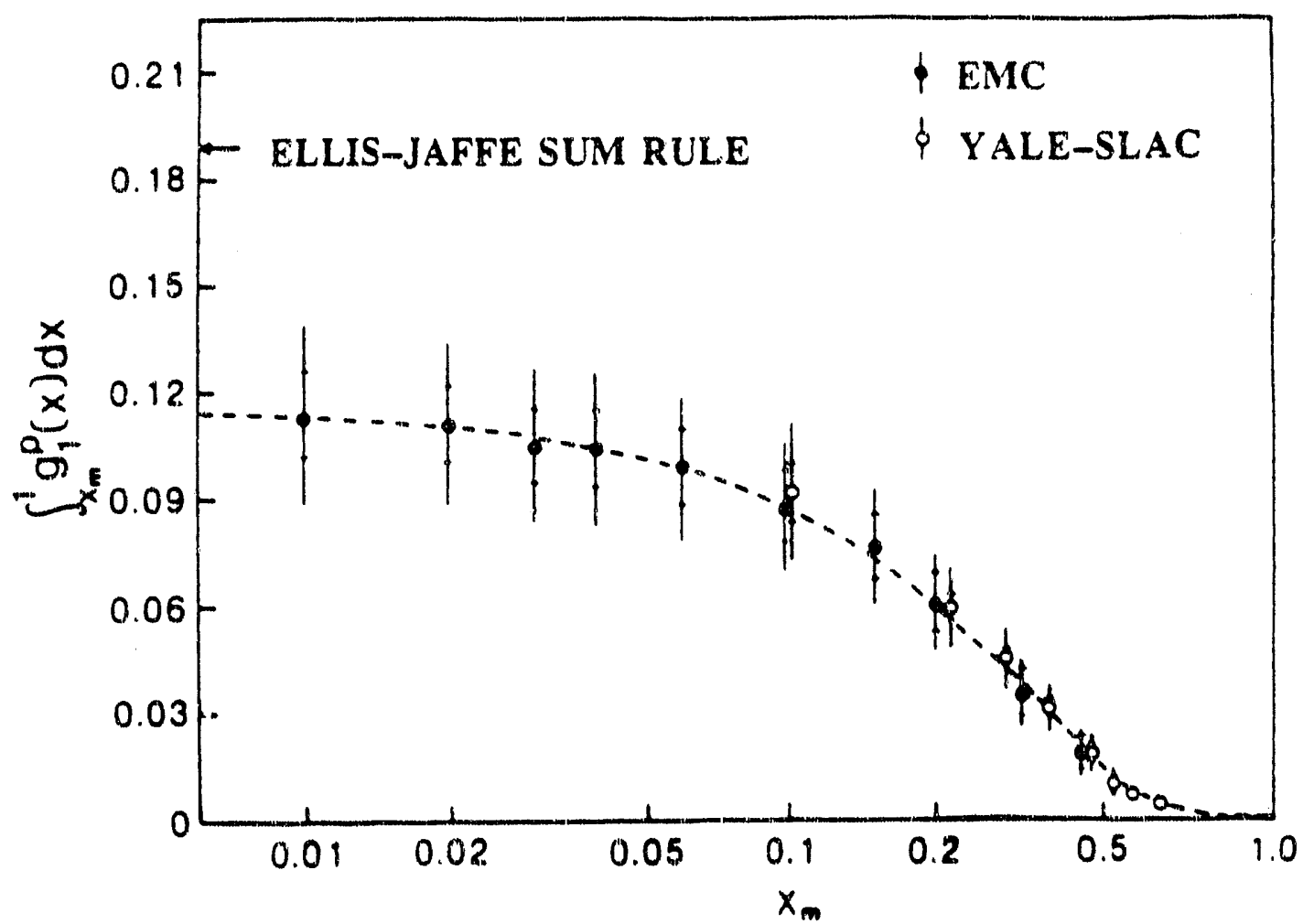

Figure 1. Integral of $g_{1}^{p}$ over $x$ as a function of the lower integration limit as obtained from the combined analysis [3] of Yale-SLAC and EMC data. The Ellis-Jaffe sum rule value is shown for comparision.

to be ruled out by the data, which extend down to $Q^{2}=0.5(\mathrm{GeV} / \mathrm{c})^{2}$ [14]. The result has also been interpreted as evidence against $Q C D$ [15]. It has also been argued that in certain Skyrme models using massless quarks and a large $1 / N_{c}$ limit $(16)$, the EMC result can be understood and that the spin of the proton is rnainly due to the angular momentim of its quarks. In a more elaborate idea the EMC result is connected to the anomaly in the singlet axial current [17]. The anomaly induces a even with $Q^{2} \rightarrow \infty$ not vanishing contribution of the gluon distribution to the singlet axial current rnatrix element

$\Delta \Sigma^{\prime}=\Delta \Sigma-N_{f} \frac{\alpha}{2 \pi} \Delta g$

where $\Delta g$ is the spin content of the gluon distribution in the same sense as $\Delta \Sigma$ is the one of the quarks and antiquarks and $N_{f}=3$ is the number of active flavours. The $E M C$ result has to be identified with $\Delta \Sigma^{\prime}$ rather than with $\Delta \Sigma$. A large fraction of the proton spin could still be carried by the quarks and antiquarks, if $\Delta g$ is about 5 (18). This big contribution of the gluons must then almost be canceled by the contribution of the angular momentum $\left\langle L_{z}\right)$ to yield finally $\frac{1}{2}$ for the proton spin

$\frac{1}{2}=\frac{1}{2} \Delta \Sigma+\Delta g+\left\langle L_{2}\right\rangle$ 
This mechanism does not explain why $\Delta g$ should be so big nor why the singlet axial current matrix element should be about zero.

Due to possible ambiguities and non-perturbative contributions the separation of the quark and the gluon contributions to the singlet axial curren matrix element has recently been criticised by several authors $[11,19-21]$. An attempt to define the gluon and quark contributions unambiguously is made in [22].

\section{NEW EXPERIMENTS}

Five new experiments have been proposed at CERN, SLAC and DESY to investigate the spin structure of the nucleons in greater detail and to test in particular the Bjorken. sum rule. They are complementary in many respects. Their characteristic parameters are summarised in Table 1. A detailed description of all experiments, except of E-143, can be found in ref. [23].

The Spin Muon Collaboration (SMC) experiment, proposed in 1988 is the only one, which has started data taking. It is an improved version of the original EMC experiment and reaches to the lowest $x$-values. This experiment is discussed in detail in section 5 .

Two experiments have been proposed for the $22.7 \mathrm{GeV}$ electron beam at SLAC, E142 [24] scheduled for the fall of 1992 and E-143 [25] foreseen for 1993. Both experiments will use a spectrometer setup in End Station A consisting of two fixed angle magnetic spectrometers at $4.5^{\circ}$ and $7^{\circ}$ with acceptances of 0.15 and $0.45 \mathrm{msr}$ respectively. Both spectrometers together cover the range $0.04<x<0.6$ for a momentum transfer of $Q^{2}>1(\mathrm{GeV} / \mathrm{c})^{2}$.

The goal of the $E-142$ experiment is the determination of the spin dependent structure function $g_{l}^{n}$. As a neutron target a pressurised 10 bar ${ }^{3} \mathrm{He}$ gas cell of $30 \mathrm{~cm}$ length will be used. The ${ }^{3} \mathrm{He}$ will be polarised to $50 \%$ by spin exchange with $\mathrm{Rb}$ vapor of high density polarised by optical pumping with a laser [26]. Due to the admixture of the Rb vapor and $\mathrm{N}_{2}$ gas needed in the pumping mechanism for quenching, the dilution factor $f$ lies between 0.09 and 0.15 depending on $x$ rather than being $\frac{1}{3}$ as for pure ${ }^{3} \mathrm{He}$. With an existing GaAs source a bearn polarisation of about $40 \%$ can be reached, which will measured to $5 \%$ accuracy with a Moller polarimeter. The beam polarisation can be changed on a pulse-by-pulse basis. A high statistical accuracy can be obtained within a few days of data taking, but the experiment is limited by systematic uncertainties. The total systematic uncertainty of 0.016 on the first moment of $g_{1}^{n}$ includes a contribution of 0.007 from the unknown $g_{2}^{n}$ which plays a more important role at low incident energies. Together with the EMC-SLAC result for the proton a test of the Bjorken surn rule to a precision of 0.03 will be achieved.

Measurements of both spin dependent structure functions $g_{1}$ and $g_{2}$ for the proton and the neutron are planned for the E-143 experiment. The solid $\mathrm{NH}_{3}$ and $\mathrm{ND}_{3} \mathrm{am}$ monia targets of $2.5 \mathrm{~cm}$ length will be polarised by Dynamic Nuclear Polarisation [27]. A ${ }^{4} \mathrm{He}$ evaporation refrigerator is to cool the target down to $1 \mathrm{~K}$. At this temperature a $5 \mathrm{~T}$ magnetic field is necessary to reach the projected polarisations of $90 \%$ and $45 \%$ 
Table 1

Characteristic parameters of the new experiments

\begin{tabular}{|c|c|c|c|c|c|c|}
\hline Target & $\begin{array}{c}\rho \\
\mathrm{cm}^{-2}\end{array}$ & $\begin{array}{l}P_{k} \\
\%\end{array}$ & $\begin{array}{l}P_{b} \\
\%\end{array}$ & $\begin{array}{c}E_{b} \\
\mathrm{GeV}\end{array}$ & $\bar{I}$ & $x$ \\
\hline
\end{tabular}

\begin{tabular}{llrrrrrr}
$S M C$ CERN & \multicolumn{1}{c}{} & taking data \\
\hline $\mathrm{C}_{4} \mathrm{H}_{9} \mathrm{OH}$ & 0.13 & $3-4.5 \cdot 10^{25}$ & 80 & 80 & $\mu 100 \cdot 200$ & $0.5 \mathrm{pA}$ & $0.005-0.7$ \\
$\mathrm{C}_{4} \mathrm{D}_{9} \mathrm{OD}$ & 0.24 & & $26-40$ & & &
\end{tabular}

E142 SLAC

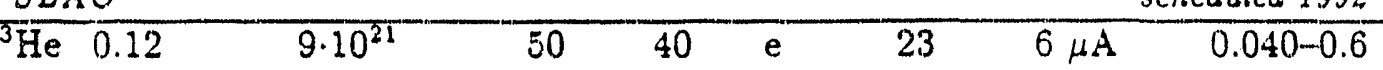

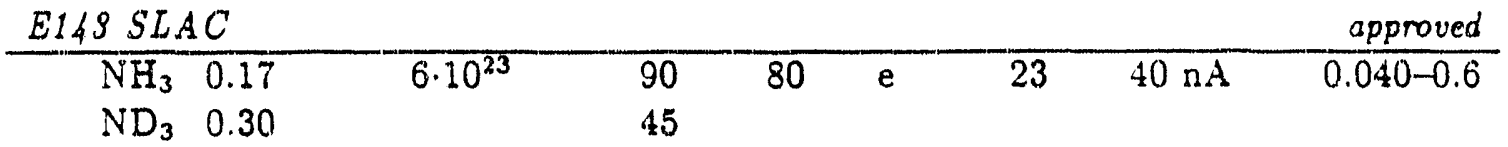

BERMES DESY

\begin{tabular}{ccccccccc} 
HERMES DESY & & & \multicolumn{3}{c}{ awaiting approval } \\
\hline $\mathrm{H}$ & 1 & $10^{14}$ & 80 & $50 ?$ & e & $30-35$ & $60 \mathrm{~mA}$ & $0.020-0.8$ \\
$\mathrm{D}$ & 1 & $2 \cdot 10^{14}$ & 80 & & & & & \\
${ }^{3} \mathrm{He}$ & 0.33 & $9 \cdot 10^{14}$ & 50 & & & &
\end{tabular}

\begin{tabular}{crrrrrrrrr} 
HELP CERN LEP & & & & & & letter of intent \\
\hline $\mathrm{H}$ & 1 & $10^{13}$ & 90 & $50 ?$ & $\mathrm{e}$ & 45 & $3 \mathrm{~mA}$ & $0.010-0.75$ \\
$\mathrm{D}$ & 1 & $2 \cdot 10^{13}$ & 60 & & & & & \\
\hline
\end{tabular}

For the ${ }^{3} \mathrm{He}$ targets the dilution factor $f$ refers to polarised neutrons, for the deuteron targets it refers to polarised deuterons. The other parameters are the target thickness $\rho$, the target and beam polarisations $P_{t}$ and $P_{b}$, the beam energy $E_{b}$ and the average beam current $\bar{I}$.

for the protons and deuterons respectively. The choice of the target material and refrigerator type is determined by the radiation resistance and the amount of heat deposed in the target. Recent developments on electron sources using strained epitaxial layers of GaAs grown on GaAsP have yielded electron polarisations of greater than $80 \%$ [28]. The relatively low current extracted from these sources would be sufficient for the requirements of the E- 143 experiment. Further progress in this field is expected before the E-143 experiment will start data taking. The planned accuracy for the test of the Bjorken sum rule is again 0.03. Using the EMC data the contribution to the error from the extrapolation to $x=0$ can be reduced.

The HERMES [29] and the HELP [30] collaborations have proposed to use internal targets in the HERA storage ring at DESY and in LEP at CERN respectively. The great advantage of internal targets is that no unpolarisable nucleons are present in the target material, so that the dilution factor is essentially $f=1$. The polarisation of the electron beam is generated by the Sokolov-Ternov effect [31]. Up to now polarisations 
of the order of $9 \%$ have been reported for both storage rings [32].

The HERMES collaboration at DESY plans the most complete set of measurements. Both spin dependent structure functions will be rneasured with internal gas targets of hydrogen, deuterium and ${ }^{3} \mathrm{He}$ and the Bjorken sum rule will be tested to an uncertainty of $0.010-0.015$, depending on which target is used for $g_{1}^{n}$. In addition the two spin 1 structure functions of the deuteron [33] will be explored. HERA provides a $30-35 \mathrm{GeV}$ electron bearn with an average current of $60 \mathrm{~mA}$. A pair of spin rotators will be installed before and behind the experiment to turn the electron spins from the transverse to the longitudinal orientation and vice versa. The beam polarisation will be measured by Compton back scattering of circular polarised laser light to 2-3\% accuracy. If a beam polarisation greater than $50 \%$ can be achieved at HERA in 1992, data taking could start in 1994. The proposed target is a windowless open storage cell, fed by a high intensity source of polarised atoms [34]. The polarisation will be reversed every few seconds. A holding field of $0.35 \mathrm{~T}$ will reduce the depolarisation due to the magnetic field of the electron bunches and to collisions with the cell walls. The target polarisation of $80 \%$ for hydrogen and deuterium will be measured on extracted atoms by a Breit-Rabi type polarimeter to $3 \%$ precision. A large solid angle forward spectrometer will detect the scattered electrons covering the range $0.02<x<0.8$.

The HELP collaboration proposes to use an internal polarised jet gas target and a large solid angle forward spectrometer at the $46.5 \mathrm{GeV}$ electron beam of LEP. The advantage of a jet target over a storage cell is that depolarisation effects are not important. On the other hand the thickness of a jet target is smaller by one to two orders of magnitude. In addition the available beam current in LEP is only $3 \mathrm{~mA}$., so that the total luminosity is smaller by at least two orders of magnitude as compared to that of the HERMES project.

\section{THE SMC EXPERIMENT}

The experiment of the Spin Muon Collaboration [35] aims at determining the spin dependent structure functions $g_{1}$ for both the proton and the neutron. In particular the Bjorken sum rule $\Gamma^{p}-\Gamma^{n}=0.191$ will be tested to 0.015 statistical and 0.018 systematical uncertainty. For the first moment of $g_{l}^{p}$ a reduction of both the systematical and the statistical errors by a factor two as compared the the EMC-SLAC result is planned. An exploratory measurement of the second spin dependent structure function $g_{2}$ of the proton is foreseen later in the schedule.

To reliably evaluate the concerned sum rules it is essential to measure the structure functions to very low values of $x$. Due to the high energy of the incoming muons, $x$ values down to 0.005 can be reached in the SMC experiment while keeping $Q^{2}$ above $1(\mathrm{GeV} / \mathrm{c})^{2}$.

In contrast to the EMC experiment, where ammonia beads were used, SMC uses beads of butanol and deuterated butanol as proton and neutron targets respectively. To generate the paramagnetic centers needed for the Dynamic Nuclear Polarisation [27] the 
butanol is doped with a metallo organic substance EHBA-Cr(V). The target consists of two oppositely polarised sections being exposed to the beam simultaneously, to minimise systematic uncertainties. Differences in the spectrometer acceptance betwee.s the upstream and downstream sections are mostly canceled by a frequent inversion of the polarisation in both sections and by averaging of the asymmetries obtained for these two configurations. The remaining uncertainties are mainly due to time dependent changes in the acceptance. This was actually found to be the dominant contribution to the systematic error in the EMC experiment. To reduce these uncertainties SMC has improved the stability of the spectrorneter by new and additional chambers and by a more frequent inversion of the target polarisation possible due to the different target material. A polarimeter has been installed to measure the polarisation of the muon beam, which in the EMC analysis has been taken from Monte Carlo studies. This will reduce another important contribution to the systematic error.

During four weeks of the 1991 run with a polarised deuteron target, sightly more data were collected than were used in the analysis of EMC. These data will allow a first look at the spin dependent structure function of the neutron. For 1992 measurements with deuteron and proton targets are foreseen. A new target is under contruction and will be used from 1993 onwards.

A more detailed description of the apparatus is given in the next sections.

\subsection{The Spectrometer}

The experiment makes use of the muon beam at the CERN-SPS. The beam line has been optimised for muons of energies up to $220 \mathrm{GeV}$ to provide a beam spot of about $4 \mathrm{~cm}$ diameter at the target. The muons originating from pion (and kaon) decay have a natural longitudinal polarisation of about $80 \%$. The open spectrometer (Fig. 2) was originally designed and constructed by the EMC [36] and was thereafter improved by the New Muon Collaboration (NMC). In the beam region the spectrometer has been adapted to the high flux of $4 \cdot 10^{7}$ muons/pulse $(\approx 2 \mathrm{~s})$. The scattered muon, whose momentum is analysed by the Forward Spectrometer Magnet, is identified by a set of trigger hodoscopes after having penetrated an iron absorber wall. To assure the stability of the tracking efficiency the aged EMC drift chambers in this region were replaced by an array of streamer and drift tubes, altogether consisting of 44 planes. Additional chambers upstream of the absorber increase the redundancy also in this region. Two low angle trigger systems, originally installed by NMC, extend the angular acceptance for the scattered muon down to $2.5 \mathrm{mrad}$ and are especially designed to accept the low $x$ events.

\subsection{The Polarised Target}

In 1991 the original EMC polarised target [37] was reinstalled and successfully put into operation with deuterated butanol. The butanol beads are contained in the two $40 \mathrm{~cm}$ long target sections of $5 \mathrm{~cm}$ diameter, which are separated by a $20 \mathrm{~cm}$ long gap. The other main parts of the polarised target are the ${ }^{3} \mathrm{He}-{ }^{4} \mathrm{He}$ dilution refrigerator [39] and the superconducting solenoid providing a longitudinal magnetic field of $2.5 \mathrm{~T}$. Dur- 


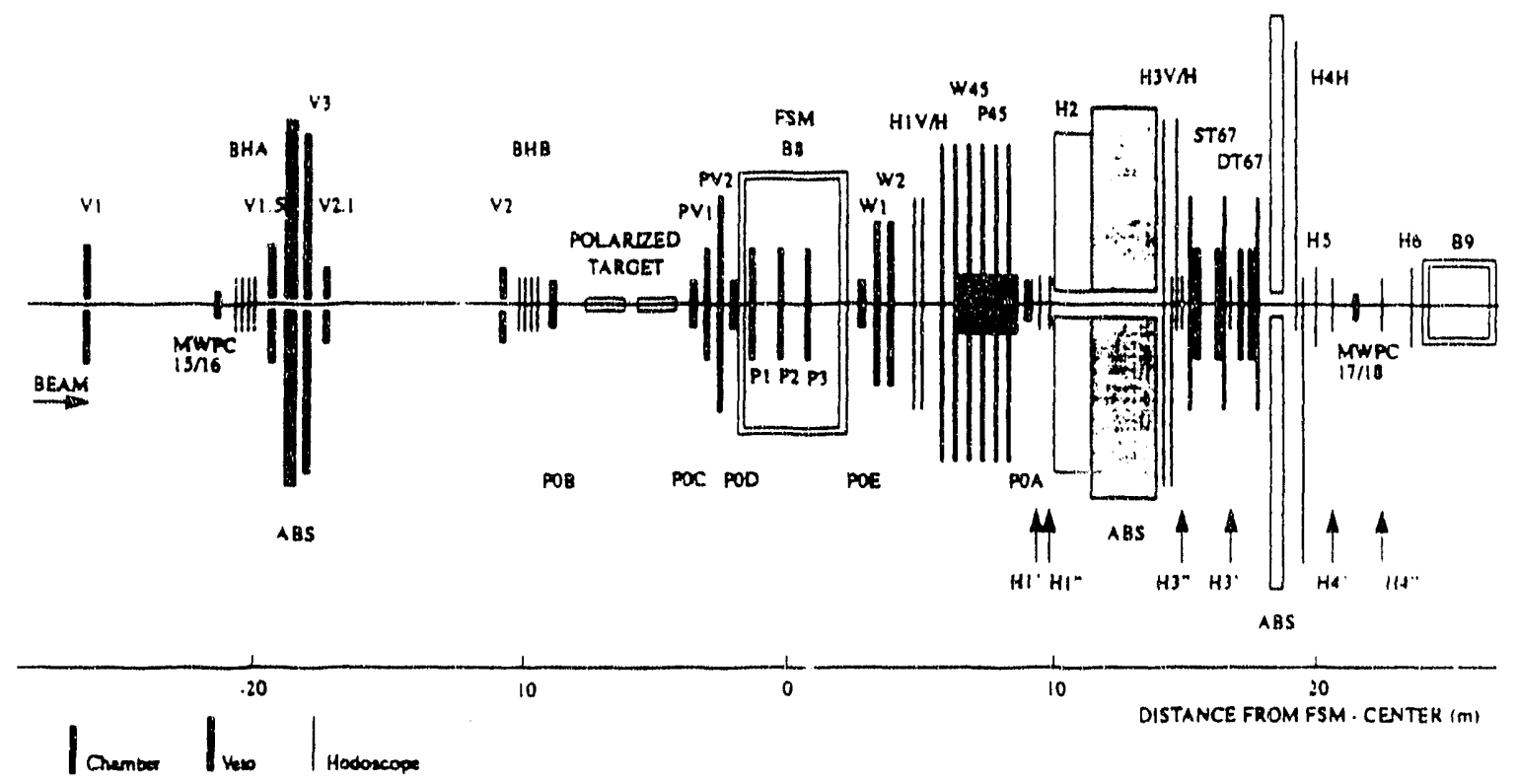

Figure 2. The SMC spectrometer. Shown are the veto counters V, the beam hodoscopes BH, proportional chambers $P$, the Forward Spectrometer Magnet FSM, drift chambers $W$, the trigger hodoscopes $H$ and the streamer and drift tube arrays ST67 and DT67.

ing dynamic polarisation the temperature in the target region is about $0.5 \mathrm{~K}$. In a simple picture under these conditions the free electron spins are completely aligned, whereas protons and deuterons are essentially unpolarised. Dynamic Nuclear Polarisation [27] transfers the electron polarisation to the protons or deuterons by means of microwave radiation close to the electron spin resonance line of $70 \mathrm{GHz}$. The slightly different frequencies needed to generate parallel and antiparallel longitudinal polarisation are provided by two separate microwave systems. This allows to built up opposite polarisations in the two target sections simultaneously. Once the maximum polarisation is reached and the microwave power is switched off, the target is cooled down to $50 \mathrm{mK}$, where the nuclear spin-lattice relaxation becomes extremely slow and the target polarisation is effectively frozen. The polarisation is measured by eight NMR-coils along the target volume with a precision of $3 \%$. Their signals were calibrated at thermal equilibrium of $1 \mathrm{~K}$, where the natural deuteron polarisation is only $0.05 \%$. If the distortion w the signal by the NMR circuit is taken into account, the deuteron polarisation can be deduced not only from the signal area but also from the asymmetry of the signal, which is split into two components due to the electric quadrupole moment of the deuteron.

In the 1991 deuterium runs the polarisation of both target sections was reversed about every 24 hours compared to about once a week during the EMC experiment. A preliminary analysis of the NMR-signals taken during these runs yields a mean polarisation of about $26 \%$ for the deuterons.

For the coming years a new polarised target with $50 \%$ longer target sections is presently under contruction. A new powerful dilution refrigerator will provide a cooling 


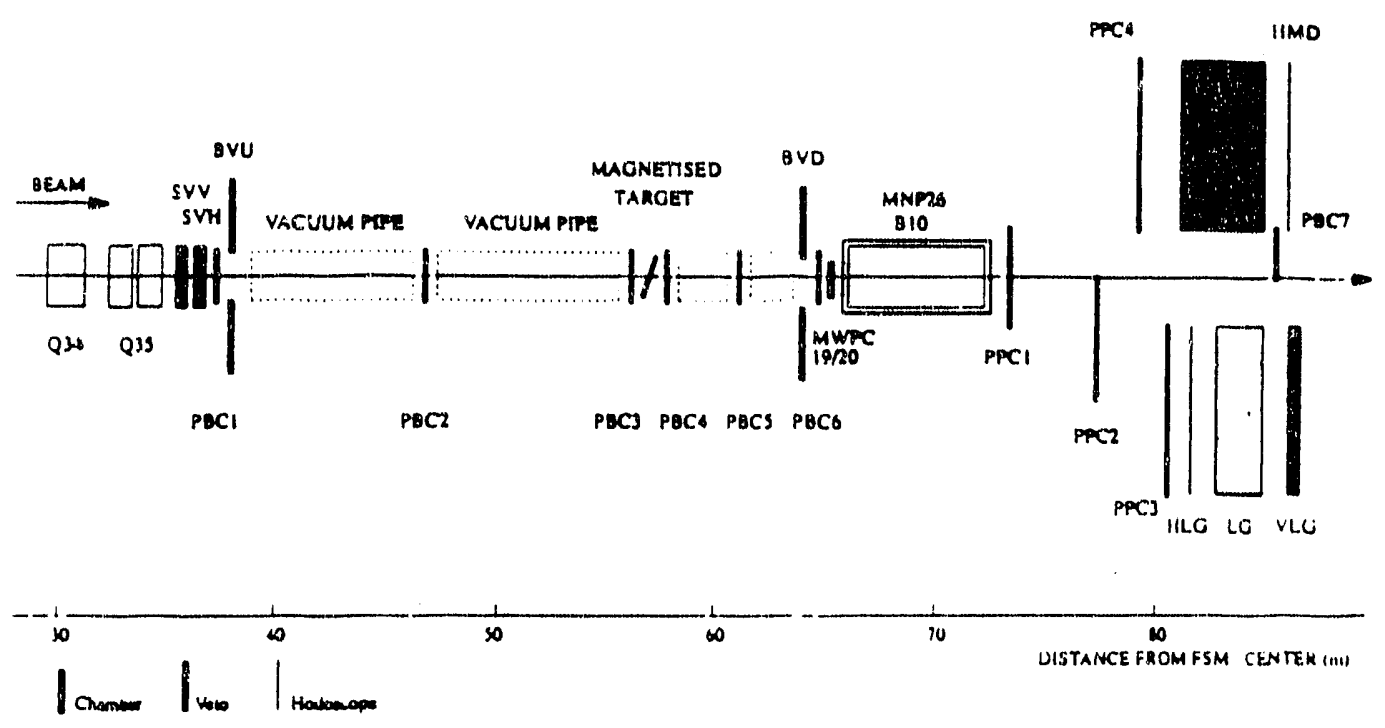

Figure 3. The SMC beam polarimeter. Shown are the shower vetos SV, the proportional beam chambers PB, the dipole magnet MNP26, the proportional chambers for scattered or decay particles PP, hodoscopes $\mathrm{H}$ and the lead glas LG.

power of $2 \mathrm{~W}$ at $0.5 \mathrm{~K}$. The new superconducting magnet system, presently being built at Saclay [38], consists of three concentrical layers. The inner solenoid will provide together with the 16 corrections coils of the middle layer a field of $2.5 \mathrm{~T}$ with a homogeneity of $|\Delta B / B|=2 \cdot 10^{-5}$ in the cylindrical target volume of $1.5 \mathrm{~m}$ length and $5 \mathrm{~cm}$ diameter. The dipole forming the outer layer provides a $0.5 \mathrm{~T}$ field allowing transverse polarisation of the target necessary for a measurement of $g_{2}$. Together with the solenoid a fast reversal of the target polarisation by the rotation of the magnetic field will become possible. For this new target a deuteron polarisation of $40 \%$ and a higher homogenity of the polarisation along the target volume is expected.

\subsection{The Beam Polarimeter}

The polarimeter (Fig. 3), which is located downstream of the main spectrometer, will determine the beam polarisation by two independent methods. The first method uses the characteristic dependence of the energy spectrum of the decay positrons on the muon polarisation. A shower veto scintillator hodoscope defines the beginning of a $30 \mathrm{~m}$ long decay distance. At its end the momentum of the decay positrons is analysed by a spectrometer magnet. The particle identification is finally done by an energy measurement in a lead glass calorimeter. This method is expected to give a statistical accuracy of $5 \%$ within 5 hours of data taking.

In the second method the scattering of beam muons from polarised electrons in a magnetised ferromagnetic iron foil is used. The polarisation is deduced from the asymmetry of two measurements with the foil magnetised in opposite directions. The recoil electron is detected in the lead glass calorimeter. In a second detection arm the positive muon is identified by a scintillator hodoscope behind an iron absorber wall on 
the opposite side of the beam line. With this method it will take about 5 times as long as with the decay method to achieve the sarne statistical accuracy. However, it is less affected by systematical uncertainties.

In both methods the tracking is done by several multi-wire proportional chambers downstream and upstream of the spectrometer magnet. A combination of both methods will result in a beam polarisation measurement of about $5 \%$ overall accuracy.

\section{CONCLUSIONS}

The interpretation of the EMC-SLAC result on the spin dependent structure function of the proton is still unsettled and new experimental input is urgently needed to make further progress. Particularly a verification of the EMC result with higher precision and a measurement of the spin dependent structure function of the neutron, which will enable us to test the fundamental Bjorken sum rule would have a big impact.

Five new experiments have been proposed at CERN, SLAC and HERA. Three of them, SMC, E-142, E-143 have been approved and SMC at CERN has started data taking in 1991 with a polarised deuteron target. For 1992 further data on $g_{1}^{n}$ are expected from SMC and the E-142 experiment at SLAC. In the years to come the experimental knowledge on the spin dependent structure function will increase enormously and help to deepen the understanding of the nucleon structure.

1 W.B. Atwood et al., Phys. Rev. D12 (1975) 1884

2 SLAC E-80, M.J. Alguard et al., Phys. Rev. Lett. 37 (1976) 1261;

Phys. Rev. Lett. 41 (1978) 70;

SLAC E-130, G. Baum et al., Phys. Rev. Lett. 51 (1983) 1135

3 EM Collaboration, J. Ashman et al., Phys. Lett. B206 (1988) 1167;

EM Collaboration, J. Ashman et al., Nucl. Phys. B328 (1989) 1

4 J.D. Bjorken, Phys. Rev. 148 (1966) 1467; Phys. Rev. D1 (1970) 1376

$5 \quad$ R. Windmolders, Int. J. Mod. Phys. A7 (1992) 639

6 R.L. Jaffe, Comments Nucl. Part. Phys. 19 (1.990) 239

7 M.G. Doncel and E. de Rafael, Nuovo Cimento 4A (1971) 363

8 L.W. Whitlow et al., Phys. Lett. B250 (1990) 193

9 NM Collaboration, I.G. Bird, proc. LP-HEP 91, Geneva, June 1991, to be published;

SLAC, L.W. Whitlow, SLAC-Report-357 (1990);

BCDMS, A. Benvenuti et al., Phys. Lett. B223 (1989) 485; B237 (1990) 592

10 J. Ellis and R.L. Jaffe, Phys. Rev. D9 (1974) 1444; D10 (1974) 1669

11 R.L Jaffe and V.A. Manohar, Nucl. Phys. B337 (1990) 509

12 F.E. Close and R.G. Roberts, Phys. Rev. Lett, 60 (1988).1471

13 M. Anselmino, B.L Ioffe and E. Leader, Sov. Nucl. Physics 49 (1989) 136

14 G. Baum et al., Phys. Rev. Lett. 45 (1980) 2000

15 G. Preparata and J. Soffer, Phys. Rev. Lett. 61 (1988) 1167

16 S.J. Brodsky, J. Ellis and M. Karliner, Phys. Let.t. B206 (1988) 309 
17 A.V. Efremov and O.V. Teryaev, Dubna preprint E2-88-287 (1988);

G. Altarelli and G.G. Ross, Phys. Lett. B212 (1988) 391

18 G. Altarelli and W.J. Stirling, Particle World 1 (1989) 40

19 A.V. Manohar, Phys. Rev. Lett. 66 (1991) 289

20 S.D. Bass et al., J. Moscow Phys. Soc. 1 (1991) 317

S. Forte, Phys. Lett. B224 (1989) 189; Nucl. Phys. B331 (1990) 1

22 G. Altarelli and B. Lampe, Z. Phys. C47 (1990) 315

23 K. Rith, Proc. 9th Symposium on High Energy Spin Physics, Bonn, Sept. 1990, Springer Verlag (1991), 198, eds. K.-H. Althoff and W. Meyer

24 E-142, R. Arnold et al., SLAC Proposal E-142, October 1989

25 E-143, R. Arnold et al., SLAC Proposal E-143, November 1991

26 T.E. Chupp et al., Phys. Rev. 36C (1987) 2244

27 M. Borghini, Phys. Rev. Lett. 20 (1968) 419

28 T. Maruyama et al., Phys. Rev. Lett. 66 (1991) 2376

29 HERMES, K. Coulter et a.., DESY/PRC 90/1, January 1990

30 HELP, G. Ballochi et al., CERN/LEPC 89-10, LEPC/M 88, 1989

31 A.A. Sokolov and I.M. Ternov, Sov. Phys. Doklady 8 (1964) 1203

32 L. Knudsen et al., Phys. Lett. B270 (1991) 97

33 P. Hoodbhoy et al., Nucl. Phys. B312 (1989) 571

34 E. Steffens, Nucl. Phys. A497 (1989) 519c

35 SM Collaboration, J. Beaufays et al., CERN proposal SPSC 88-47, December 1988

36 EM Collaboration, O.C. Allkofer et al., Nucl. Instrum. and Methods 179 (1981) 445

37 S.C. Brown et al., Proc. 4th International Workshop on Polarized Target Materials and Techniques, Bonn, 1984, 102, ed. W. Meyer

38 A. Dael et al., CEN Saclay Preprint Cryomag/91-05 (1991), to be published in Proc. MT12, Leningrad, June 1991

39 T.O. Niinikoski, Nucl. Instrum. and Methods 192 (1982) 1.51 

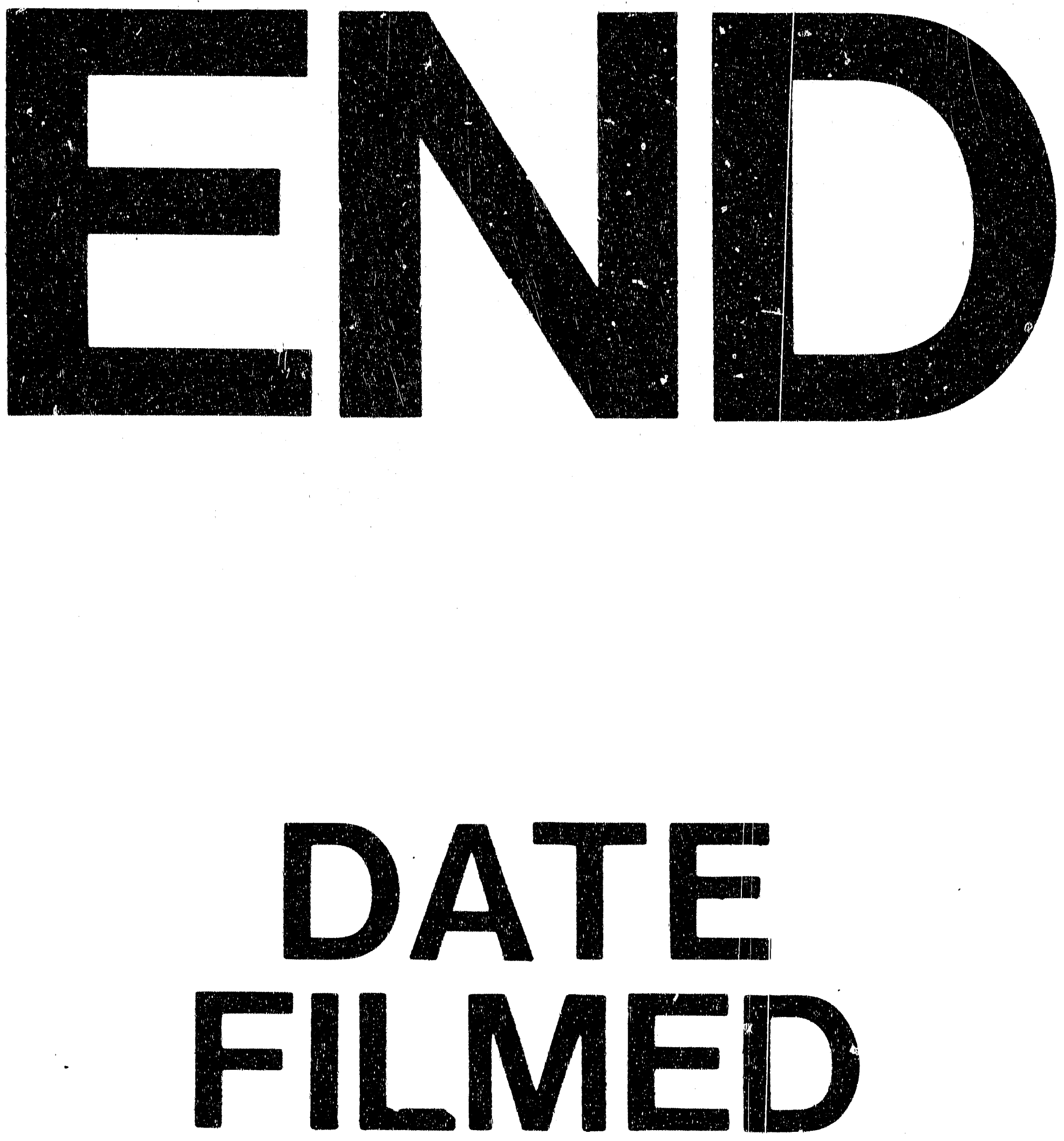

妻

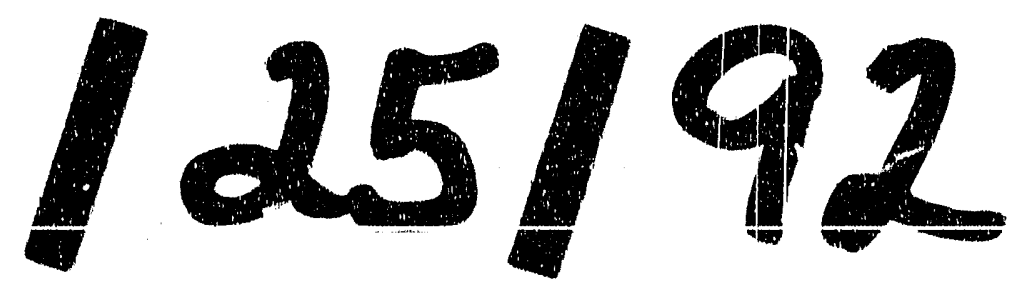


